\title{
Submitted: 19.08 .2019
Duplicated inferior vena cava with coexisting multiple Accepted: 11.09.2019 vascular anomalies and their clinical implications: a case report
}

Published: 30.09.2019

Keywords

inferior vena cava, vascular anomalies, renal vein, hepatic vein

\author{
Cezary Gołąbek¹, Karolina Druć ${ }^{1}$, Zuzanna Dusińska¹, Jakub Franke¹, \\ Emil Głowacki', Agata Kuskowska¹, Adrianna Mróz¹, Oliwia Opacka, \\ Oktawia Pięta1, Anna Świderska1, Karol Welc¹, Dominika Jaguś², \\ Agnieszka Krauze², Maciej Jędrzejczyk²
}

'Students' Research Club at the Department of Diagnostic Imaging, Second Faculty of Medicine, Medical University of Warsaw, Mazovian Bródno Hospital, Warsaw, Poland ${ }^{2}$ Department of Diagnostic Imaging, Mazovian Bródno Hospital, Warsaw, Poland

Correspondence: Dominika Jaguś, Zakład Diagnostyki Obrazowej, Mazowiecki Szpital Bródnowski, ul. Kondratowicza 8, 03-242 Warszawa; tel.: +48 2232658 10, fax: +482232659 91, e-mail: jagus.dominika@gmail.com

DOI: $10.15557 / J o U .2019 .0035$

\section{Abstract}

The paper presents the case of a 23-year-old man with multiple venous anomalies. The abnormalities were asymptomatic, and they were detected accidentally on routine abdominal ultrasound examination. The anomalies were found in the inferior vena cava, right testicular vein, left renal vein, and hepatic veins. Familiarity with different developmental variants within the inferior vena cava and other venous vessels plays a crucial role in ultrasound imaging. Vascular anomalies, although rare, should be taken into account in the differential diagnosis of focal lesions within the abdominal cavity. Also, variation in vascular anatomy may be a precipitating factor for blood flow disorders, and hence predispose patients to deep vein thrombosis and other pathological conditions. The reported case serves as a valuable addition to the knowledge of the vascular system that radiologists use in their everyday practice when performing diagnostic ultrasound examinations. 


\section{Introduction}

Anomalies of the inferior vena cava (IVC) are relatively rare. They should be detected before scheduled urological or vascular surgery or organ transplant operations because of the risk of complications. Additionally, IVC anomalies may be mistakenly diagnosed as enlarged aortic lymph nodes and unnecessarily biopsied ${ }^{(1-3)}$.

The embryogenesis of the IVC involves three pairs of cardinal veins: posterior, inferior and superior ${ }^{(3,4)}$. The complexity of embryogenesis determines the formation of various developmental anomalies within the IVC and its tributaries $^{(4)}$. The most common and clinically significant abnormalities include double IVC, left-sided (displaced) IVC, absence of the subrenal segment of the IVC, multiple renal veins, left renal vein encircling the aorta, retroaortic left renal vein, IVC draining into the azygos vein, and right ureter encircling the $\operatorname{IVC}^{(4-7)}$.

IVC anomalies usually coexist with other venous abnormalities, which are usually asymptomatic. Furthermore, vascular defects may be accompanied by organ defects such as renal agenesis, horseshoe kidney, exstrophy of the cloaca, dextrocardia or polysplenia ${ }^{(5,8)}$.

\section{Case report}

A 23-year-old man reported for a routine ultrasound examination of the abdominal cavity at the Department of Diagnostic Imaging, Second Faculty of Medicine, Medical University of Warsaw. The examination was performed using a Philips Epiq 5 ultrasound system with a convex probe 5-2 $\mathrm{MHz}$. Multiple uncommon anomalies in the venous pattern were visualised. Prior to the examination the patient had not reported any complaints or chronic diseases. The patient's family history of congenital defects or anatomical variation in the circulatory system was negative.

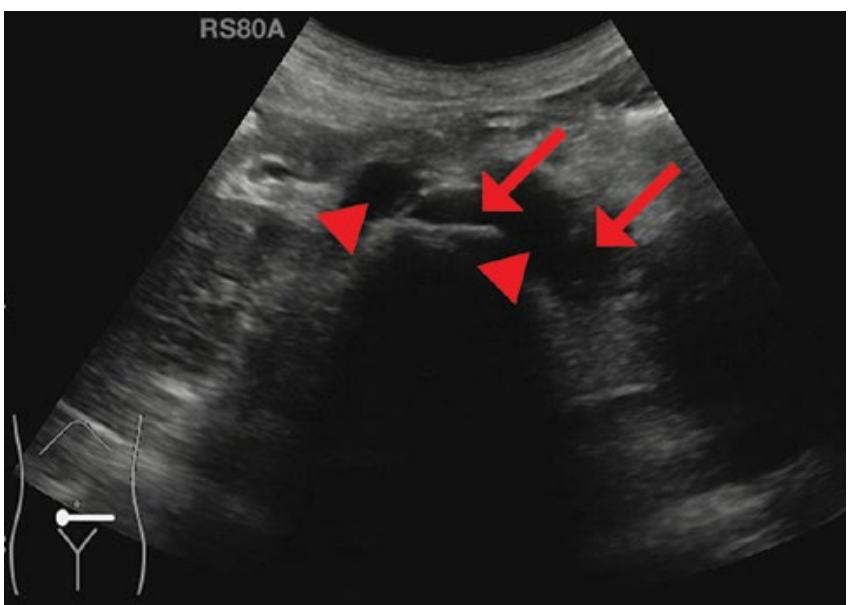

Fig. 1. Confluence of common iliac veins (marked with arrows) to the left of the aorta (invisible) and the spine. Arrowheads mark the homonymous common iliac arteries

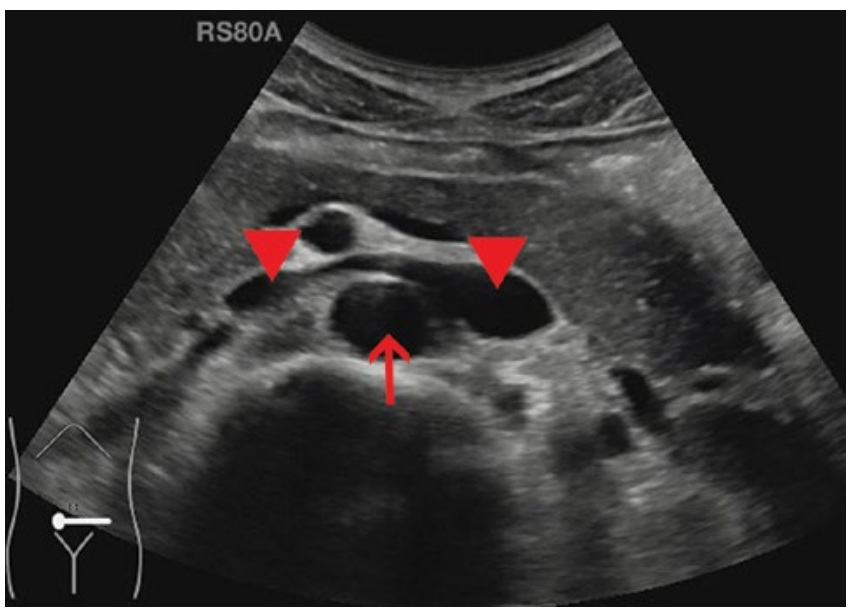

Fig. 2. IVC with left-side course crosses the aorta (arrow) anteriorly and passes to the right (course marked with arrowheads)

Ultrasound B-mode imaging revealed atypical double course of the IVC, with the dominant venous trunk on the left (LIVC). A Doppler ultrasound examination showed normal flow spectrum in the dominant LIVC. The confluence of common iliac veins was left to the aorta (Fig. 1), and passed into the LIVC with maximum transverse dilatation to $21 \times 27 \mathrm{~mm}$ just before the junction with the aorta. At the level of branching of the renal arteries from the aorta, the LIVC - crossing the aorta anteriorly - crossed to the right (Fig. 2). There, it joined the short trunk of the IVC with a right-sided course (RIVC). The further course of the IVC was normal, draining into the right atrium.

The testicular vein was visualised to the right of the aorta (Fig. 3). It had the maximum transverse dimension of $8 \times 11 \mathrm{~mm}$, joining the right renal vein below the origin of the right renal artery. Both vessels formed the vascular trunk of the RIVC into which the LIVC emptied. Additionally, the ostia of small venous vessels - probably the right lumbar veins and some mesenteric vein branches - were seen in the initial section of the testicular vein.

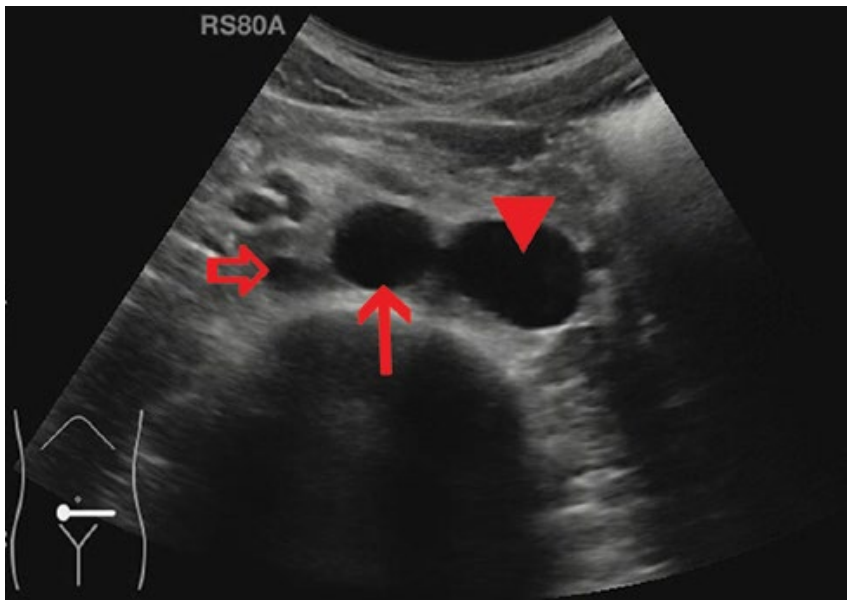

Fig. 3. IVC (arrowhead) passes to the left of the aorta (arrow). Right testicular vein (open arrow) runs along the aorta on the right 


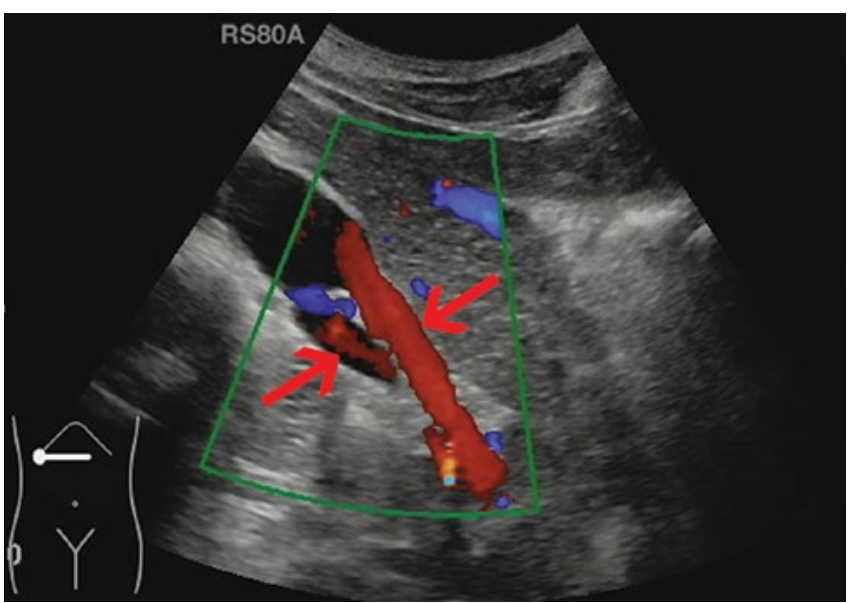

Fig. 4. Duplicated left renal vein (arrows)

The ultrasound scan also showed duplication of the left renal vein. Both branches were located along the left renal artery and drained into the LIVC, approximately $20 \mathrm{~mm}$ from its junction with the aorta (Fig. 4). The right renal vein was single, and had a normal course.

Variation was also noted in the hepatic vein system. The right and middle hepatic veins were duplicated. The left hepatic vein was triplicated or had an additional large component reaching the external branch. All these branches formed a short common trunk draining into the IVC (Fig. 5),

\section{Discussion}

The reported case describes duplication of the inferior vena cava coexisting with multiple anomalies of the renal and hepatic veins. Most typically, IVC anomalies are diagnosed by accident in patients who do not present any symptoms. However, such anomalies are often considered as a potential cause of deep vein thrombosis due to slow vascular flow and numerous collateral vessels. In addition, there is a risk of varicose veins due to the compression of anomalously positioned venous vessels ${ }^{(1)}$.

The incidence of duplicated IVC ranges between $0.2 \%$ to $3.0 \%$. The anomaly develops as a result of the left superior cardinal vein failing to regress during gestation. The anomaly presents as two veins positioned on both sides of the aorta, uniting into a single vein at the level of the renal arteries. It is especially important in patients undergoing inferior vena cava filter implantation to trap blood clots and prevent pulmonary embolism. Left-sided IVC $(0.2-0.5 \%)$ initially runs to the left of the aorta, and then crosses it - usually anteriorly - at the level of the renal arteries. Consequently, it can compress the celiac trunk and the superior mesenteric artery, resulting in the development of so-called abdominal angina. In this variant, the course of the gonadal and adrenal veins mirrors the system with the rightsided positioning of the IVC. Both IVC duplication and left-sided IVC may be erroneously diagnosed as periaortic lymphadenopathy, and vascular biopsy may be performed unnecessarily. The IVC continuing into the azygos vein (found in approximately $0.6 \%$ of cases) is a result of disorders in the formation of anastomosis of the right superior cardinal vein and the hepatic vein system. As a consequence, the hepatic segment of the IVC fails to develop, and blood flows directly from the anastomosis of the superior and inferior cardinal veins into the azygos vein which, in this pattern, drains normally into the superior vena cava. The right ureter encircling the IVC is an effect of the right superior cardinal vein failing to develop, and the persistence of the right posterior cardinal vein. In this anomaly, the right ureter encircles the IVC, crossing it initially from the right and posteriorly, and then, descending, from the left and anteriorly $(1,3-5,7)$.

Renal vein anomalies are among the most common venous abnormalities and they are usually detected by accident. However, their presence should be taken into account in a number of clinical situations, for example during catheterisation of renal vessels, testicular vein embolisation, renal and adrenal venography - or kidney transplantation ${ }^{(9)}$. Multiple renal veins are the most common anomaly occurring in the general population $(15-30 \%)$. It is observed primarily in the right renal vein (RRV) and presents as two to four veins running separately or a single vein dividing right before draining into the IVC ${ }^{(6)}$. The left renal vein (LRV) encircling the aorta affects $1.5-8.7 \%$ of the population and develops when the dorsal branch of the anastomosis between the superior and inferior cardinal veins fails to regress during embryogenesis. Retroaortic left renal vein $(1.2-2.4 \%)$ arises when, unlike in the normal process, the ventral branch disappears, leaving the dorsal branch of the above-mentioned anastomosis ${ }^{(4,8)}$.

A high degree of variation is found in the hepatic vein system. Most typically, in $95 \%$ of cases, the middle and left hepatic veins form a common trunk, while the right hepatic

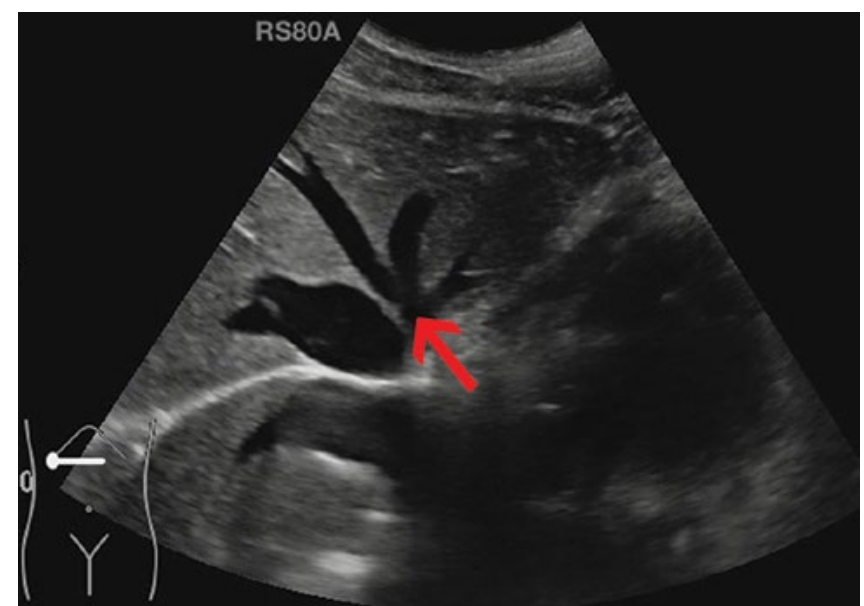

Fig. 5. Triplicated left hepatic vein (confluence of the branch marked with an arrow) 
vein drains into the IVC directly. In the remaining $5 \%$ of cases, each of the veins drains into the IVC separately. In addition, there are numerous variants in the course and tributaries of each of the hepatic veins. The pattern of hepatic veins plays a role in surgical procedures including liver transplant or liver tumour resection. When planning hemihepatectomy, i.e. surgical resection of part of the liver for the purpose of transplantation, the cutting plane should be planned in such a manner as to ensure that the hepatic veins running along it remain intact. Otherwise, there is a risk of congestive infarction and graft failure. The orientation of the cutting plane is determined primarily by the tributaries to the middle hepatic vein ${ }^{(10)}$.

\section{Conclusions}

Anatomical variation in the IVC and its tributaries may have multiple important effects and should be taken

\section{References}

1. Malaki M, Willis A, Jones R: Congenital anomalies of the inferior vena cava. Clin Radiol 2012; 67: 165-171.

2. Lohr JM, Mouawad NJ: Venous Anatomy, Development, and Variations. Current Management of Venous Diseases. Springer 2018: 3-22.

3. Eldefrawy A, Arianayagam M, Kanagarajah P, Acosta K, Manoharan M: Anomalies of the inferior vena cava and renal veins and implications for renal surgery. Cent European J Urol 2011; 64: 4.

4. Giordano JM, Trout HH 3rd: Anomalies of the inferior vena cava. J Vasc Surg 1986; 3: 924-928.

5. Bass JE, Redwine MD, Kramer LA, Huynh PT, Harris JH Jr: Spectrum of congenital anomalies of the inferior vena cava: cross-sectional imaging findings 1 (CME available in print version and on RSNA Link). Radiographics 2000; 20: 639-652.

6. Urban BA, Ratner LE, Fishman EK: Three-dimensional volume-rendered CT angiography of the renal arteries and veins: normal anatomy, variants, and clinical applications. Radiographics 2001; 21: 373-386. into account when performing surgical operations, e.g. nephrectomy, adrenalectomy, hepatectomy, and interventional radiology procedures. Anomalies should be identified in order to avoid accidental vascular injury. If a vascular anomaly is suspected, CT angiography or MRI with $3 \mathrm{D}$ reconstruction should be performed, so that detailed planning of the procedure is possible ${ }^{(3)}$.

\section{Conflict of interest}

The Authors do not declare any financial or personal links with other persons or organisations that might adversely affect the content of this publication or claim any right to the publication.
7. Geley TE, Unsinn K, Auckenthaler T, Fink CJ, Gassner I: Azygos continuation of the inferior vena cava: sonographic demonstration of the renal artery ventral to the azygos vein as a clue to diagnosis. AJR Am J Roentgenol 1999; 172: 1659-1662.

8. Truty MJ, Bower TC: Congenital anomalies of the inferior vena cava and left renal vein: Implications during open abdominal aortic aneurysm reconstruction. Ann Vasc Surg 2007; 21: 186-197.

9. Pallangyo P, Lyimo F, Nicholaus P, Masatu S, Janabi M: Bilateral anomalous drainage of the posterior divisions of renal veins into the azygos venous system in a 20-year-old woman: a case report. J Med Case Rep 2016; 10: 344 .

10. Catalano OA, Singh AH, Uppot RN, Hahn PF, Ferrone CR, Sahani DV: Vascular and biliary variants in the liver: implications for liver surgery. Radiographics 2008; 28: 359-378. 